\title{
Evaluation of Thermal Bridges Using Online Simulation Software
}

\author{
Ligia Moga ${ }^{1, *}$, Ioan Moga ${ }^{1}$ \\ ${ }^{1}$ Technical University of Cluj-Napoca, Civil Engineering and Management Department, 400027 Cluj-Napoca, Romania
}

\begin{abstract}
In order to reach nearly Zero Energy Buildings, a thorough design must be given in designing proper junctions, which will overall reduce the impact of the thermal bridges on the thermal performance of the building envelope. It is well-known that a thermal bridge is a weak thermal area of the building envelope through which increased heat losses occur. For the thermal bridges' evaluation, several numerical simulation software exist on the market, but their usage implies knowledge regarding the numerical modelling and simulation using various numerical methods (i.e. finite element method, finite difference methods, and others). Due to time constraint, designers use thermal bridges atlases that provide values for the linear heat transfer coefficient for several types of thermal bridges. Nevertheless, the multitude of existing thermal bridges requires more and more atlases which are not feasible in elaborating, due to time constraints. In order to respond to this demand, the authors developed a software for the modelling and simulation of thermal bridges that can be easily accessed by practitioners. The paper presents the software its components and the way that the user can interact with it.
\end{abstract}

\section{Introduction}

According to the latest European directives on energy performance of buildings [1-2], starting from 31 December 2018 all public buildings, and from 31 December 2020 all new buildings must met the requirements of nearly Zero Energy Buildings (i.e. nZEB). Furthermore, based on the requirements of the last energy performance of buildings Directive (EU) 2018/844 [2], the same nZEB levels defined for new buildings, must also be met by existing buildings.

The definition of nZEB can vary from country to country [3], from nearly zero energy building, to zero energy building, net zero energy and zero energy, but also zero carbon and zero net carbon. No matter how the building is defined, a proper thermal design of the building envelope is one of the key points in obtaining such a high performant energy efficient level. A proper design implies the modelling and simulation of all weak thermal areas of the building envelope, denoted as a "thermal bridge area". The assessment of thermal bridges can be made by using free or commercial available software for modelling and simulation, e.g. CÎMPSPAT, THERM, DarTWin, KOBRA, TRISCO, PHYSIBEL, HTFlux and others. These software are able to do twodimensional or three dimensional calculations of the temperature fields, using several numerical methods. Due to time constraints, specialist also use thermal bridges atlases that describe typical constructive details met in current practice [4]. There are also available standards that give values for the thermal bridges assessment, the most known being the European Standard ISO 14683 [5].
Although that the standard contains a large number of cases, i.e. around 66 cases that refer to eight types of thermal bridges (exterior walls, interior walls, wall corners, intermediate floors connection, windows and doors connection with the opaque area, roof connections, slab-on-ground and others), the described details do not cover all the existing constructive details from the European market.

Despite the fact that several options are found on the market, practitioners have a hard time in either finding the required thermal bridges. Another issue is due to the lack of knowledge in modelling and simulating such cases. In several situations, practitioners just choose a reduction coefficient for the thermal transmittance of the building component, without assessing the problematic joints. This approach leads to inaccurate results in evaluating the energy performance of the building. As mentioned by Kosny [6] the unidirectional thermal transmittance defined based on the component structure represents about $50 \%$ to $80 \%$ of the entire area of the element, the rest being the thermal bridges area. Also, as mentioned by Erhorn-Kluttig [7] the impact of thermal bridges on the annual energy consumption for heating can go around $30 \%$ and even increase in the case of building retrofit. Consequently, thermal bridges can't be substituted with simplified calculation which will deviate from the required $n Z E B$ path.

For the purpose of helping the construction market, the authors developed an online software for the modelling and simulation of thermal bridges. The paper presents the main aspects of the online tool and the manner in which a practitioner can interact with it. The software contains an

* Corresponding author: ligia.moga@ccm.utcluj.ro 
extensive database of thermal bridges, based on which the user can build (i.e. elaborate) in a quick and reliable way other cases.

The database of the program contains details for all types of joints currently met at building design in east European countries, with a focus on the Romanian practice. The software is addressed to all types of users, starting from the ones with proper knowledge regarding thermotechnical calculations, but also for the ones that are beginners in this field. The novelty of the software results from the fact that the user free of modelling and simulation skills, can interact with the program. The interaction requires just standard computer skills, as accessing the platform, choosing the type of thermal bridge, adjusting the input parameters, and executing the case.

\section{Methodology}

The European standard EN ISO 10211[8], defines a thermal bridge as being one or all the following situations: - $\quad$ area in the building envelope that is penetrated by materials that have different thermal conductivity, or/and - changes in the dimension of the building envelope, or/and

- changes exist between interior and exterior areas. Considering the above mentioned situations, thermal bridges are defined, as it follows:

- linear thermal bridge: is characterised by a linear heat transfer coefficient $\psi[\mathrm{W} /(\mathrm{m} . \mathrm{K})]$

- $\quad$ point thermal bridge: is characterised by a point heat transfer coefficient $\chi[\mathrm{W} / \mathrm{K}]$

The assessment of a thermal bridge is done following the prescription of the European standards EN ISO 10211 [8]. In order to calculate the thermal transmittance of a building component, one needs to know the value of the linear heat transfer coefficient, defined by $\psi[\mathrm{W} /(\mathrm{m} \cdot \mathrm{K})]$ :

$$
\psi=L^{2 D}-U_{j} \cdot l_{j}
$$

where: $\mathrm{L}^{2 \mathrm{D}}$ is the two-dimensional thermal coupling coefficient, $\mathrm{Uj}$ the unidirectional thermal transmittance, $\mathrm{lj}$ is the length of the two-dimensional geometrical model over which the $\mathrm{Uj}$ value is applied.

Knowing the $\psi$ value, the thermal transmittance $U$ ' $\left[\mathrm{W} /\left(\mathrm{m}^{2} \cdot \mathrm{K}\right)\right]$ of the component, is calculated:

$$
U^{\prime}=\sum_{i} A_{i} \cdot U_{i}+\sum_{k} l_{k} \cdot \psi_{k}+\sum_{k} \chi_{k}
$$

where: A is the area of the assessed element of the building envelope, $l_{k}$ is the length over which the linear heat transfer coefficient is applied, $\chi$ is the point thermal transmittance obtained through a 3D simulation.

The unidirectional thermal transmittance is calculated following the formula:

$$
U=\left[R_{s i}+\sum \frac{d}{\lambda}+R_{s e}\right]^{-1}
$$

where: Rsi and Rse are the thermal resistances of the superficial air layers (i.e. interior and exterior) in $\left[\mathrm{m}^{2} \cdot \mathrm{K} / \mathrm{W}\right], \mathrm{d}$ thickness of the layer of material in $[\mathrm{m}]$ and $\lambda$ the thermal conductivity of the material in $[\mathrm{W} /(\mathrm{m} \cdot \mathrm{K})]$.

The online version of the PSIPLAN [9] modelling and simulation software, is based on solving the plane heat transfer differential equation in steady state thermal regime (see 4 ), where $\theta$ is the temperature in the node $(\mathrm{x}, \mathrm{y})$, and $\lambda(\mathrm{x}, \mathrm{y})$ has constant valued for the materials describing the detail.

$$
\frac{\partial}{\partial x} \cdot\left[\lambda(x, y) \cdot \frac{\partial \theta(x, y)}{\partial x}\right]+\frac{\partial}{\partial y} \cdot\left[\lambda(x, y) \cdot \frac{\partial \theta(x, y)}{\partial y}\right]=
$$

Boundary conditions are set for internal and external environment in accordance to national and European standards. The discretization is done following the finite difference method. The temperature field is obtained in the nodes of the spatial discretization network of the thermal bridge detail. The program considers the stipulations of the standard EN ISO 10211 [8], for the discretization network, and the estimation of the flows equilibrium, in the nodes of the network. The digitization network is done automatically by the program, thus making a user friendly program that requires simple operation of it. The meshing is performed automatically, until the conditions for the heat flow between the inner and outer surfaces of the wall end up with a difference under $0.001 \mathrm{~W}$. Additionally, in each node of the spatial mesh the obtained differences is under $0.000001 \mathrm{~W}$. [8]

The data input is done in a graphical way by using a graphical module. As input data the program uses the spatial geometry of the component, physical characteristics of the materials constituting the building envelope component, the contour conditions for the superficial resistances, the ambient temperatures, the exterior temperature, and the interior and exterior air humidity. Climatic data specific for the reference locations in accordance with the SR EN ISO 13790 standard [10] and other specific standards, e.g. the exterior air temperature, the exterior air humidity, the atmospheric pressure, the direct and diffuse solar radiation by orientations and the direction of dominant winds, are contained in the library of the program. The programming language used for the program was developed from Fortran to Pascal and up to Delphi, having inserted the calculation modules in $\mathrm{C}++$ language.

As it was mentioned in our previous papers $[4,11]$, the modelling software is based on the standard prescription regarding the calculation of the linear thermal transmittance $\psi[\mathrm{W} /(\mathrm{m} \cdot \mathrm{K})]$ and the temperature factor $\mathrm{f}_{\mathrm{Rsi}}$ known as the condensation resistance factor.

$$
f_{R s i}=\frac{\theta_{s}-\theta_{e}}{\theta_{i}-\theta_{e}}
$$

where: $\theta_{\mathrm{s}}$ is the superficial temperature, $\theta_{\mathrm{i}}$ is the interior air temperature and $\theta_{\mathrm{e}}$ is the exterior air temperature.

Although that the methodology of calculating thermal bridges is present around Europe, there are several alternative approaches in the European building codes. Kuusk et al [12] had summarized the alternative approaches found across several European countries: 
- Belgium: the method is based on simple rules that do not require the calculation of the $\psi$ value;

- Cyprus, Austria, Greece and Spain: the calculation software is using tabulated values defined for several thermal bridges, without the need of user input;

- Estonia: the legislation gives permission of use for tabulated data, if not other information is given;

- France: it is imposed the use of an energy calculation software for thermal bridge assessment;

- Romania: the definition for the thermal transmittance takes into account both linear and point transmission. However, in the case that no values are given for the point thermal bridge, the average $U$ value will be calculated without it.

In the Romanian case, the norm C107 [13] gives the technical prescriptions for the modelling and simulation of thermal bridge. The prescription are based on the European standard [6], with some differences in the modelling length compared to the ones defined by [6]. Designers or energy auditors can use for thermal bridge assessment, the norm series $\mathrm{C} 107 / 3$ and $\mathrm{C} 107 / 5$. The two design norms details for buildings mostly built before ' 95 . Designers can also use newer thermal bridges atlases like [4] and [14], or other thermal bridges catalogues tailored for constructive details used in design activity. Once the linear and point thermal bridges values are applied, the thermal transmittance is calculated and compared to normed value. The Order 2641 [15] gives the maximum admitted normed values for residential buildings-Table 1 and for non-residential buildings, category 1-table 3 and category 2-Table 4. Even though that the Romanian market offers around ten thermal bridges atlases with various details of thermal bridges, practitioners still encounter situations when the assessed thermal bridge is not found in any of the existing catalogues. Therefore, they must use a software adequate for the modelling and simulation of the thermal bridges.

\section{The Online Working Platform}

In order for the online software to be useful in practice, a set of constructive building details were developed. The details are constituting the database of solutions, which also works as a thermal bridges catalogue.

As demonstrated in figure 1 , the user accesses the database (i.e. catalogue) of several predefined details of thermal bridges. Each case of a thermal bridge, as it can be seen in figure $1-\mathrm{a}$, includes at least fifteen preestablished cases, as it can be seen in figure 1-b, i.e. reinforced concrete column in exterior wall. Based on the chosen class of thermal bridge, the user will access the suitable thermal bridge constructive detail.

The user choses one of the predefined cases, and manipulates it, by redefining each input parameters. The characteristics, i.e. geometric dimensions, material type, thermal characteristics, glazing areas, boundary conditions and others, can be modified in accordance to the required detail. Upon setting the parameters, the detail is executed and the modelling length is automatically generated, in accordance to European design standards [8].

In spite of the fact that the user does not need knowledge regarding modelling issue in order to interact with the software, the accuracy of the results respect the convergence conditions. The software will execute the modelling and the simulation according to the standard stipulations, after the new thermal bridges is defined. The software generates the design data, the unidirectional thermal resistance $R$ (where $U=1 / R$ ), the linear thermal transmittance $\psi$ and temperature factor $\mathrm{f}_{\mathrm{Rsi}}$ for the risk of mould growth and risk of condensation.

The software has an open structure that allows other details to be added in future, by developing a new thermal bridge case starting from an existing detail. As it was previously mentioned, the database is focused on details of constructive solutions met on the Romanian construction market. The predefined details are typical for reinforced concrete frame system with masonries (e.g. as it can be seen in figure 1), reinforced concrete structural walls and some for wood based constructive solutions. 


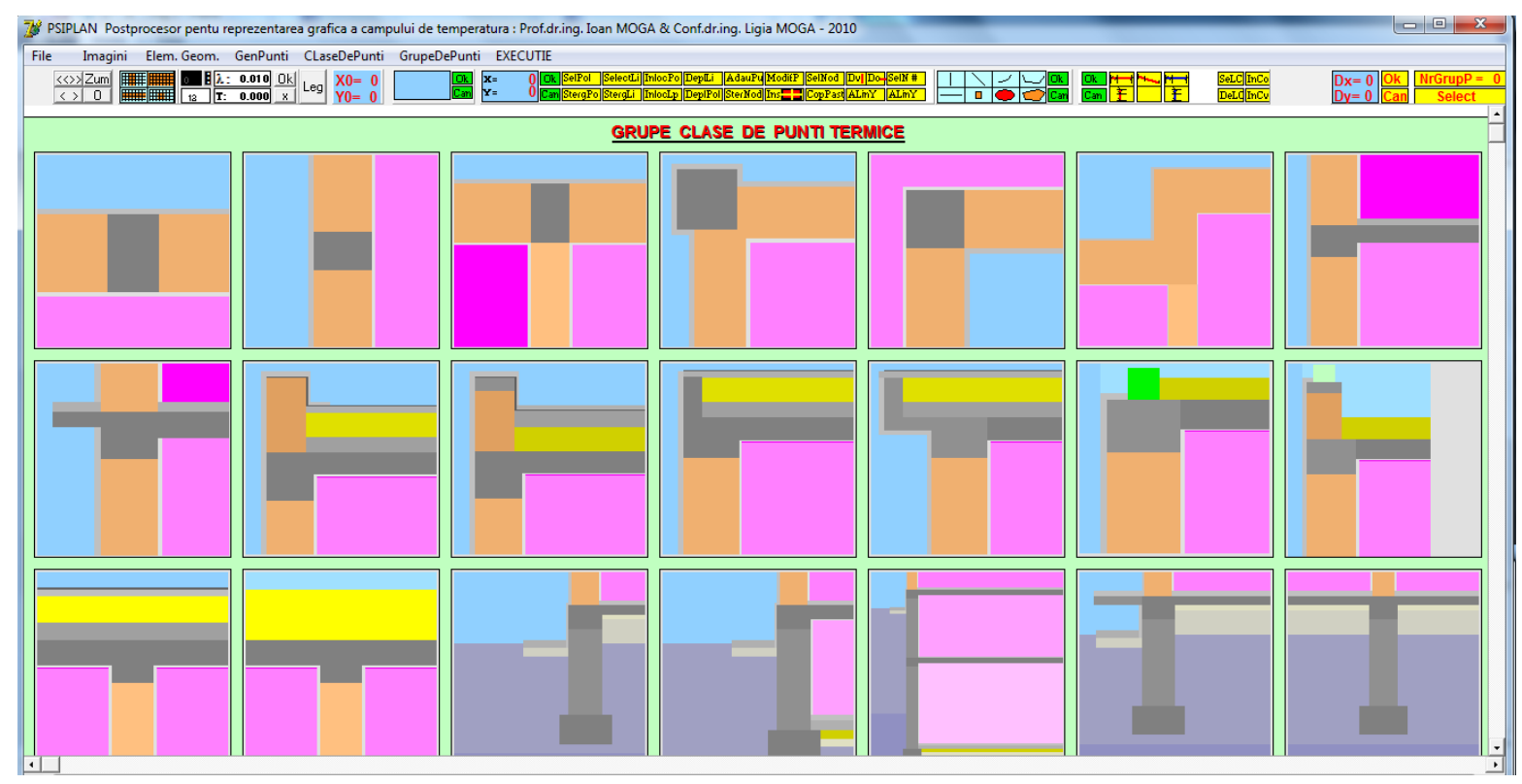

(a)

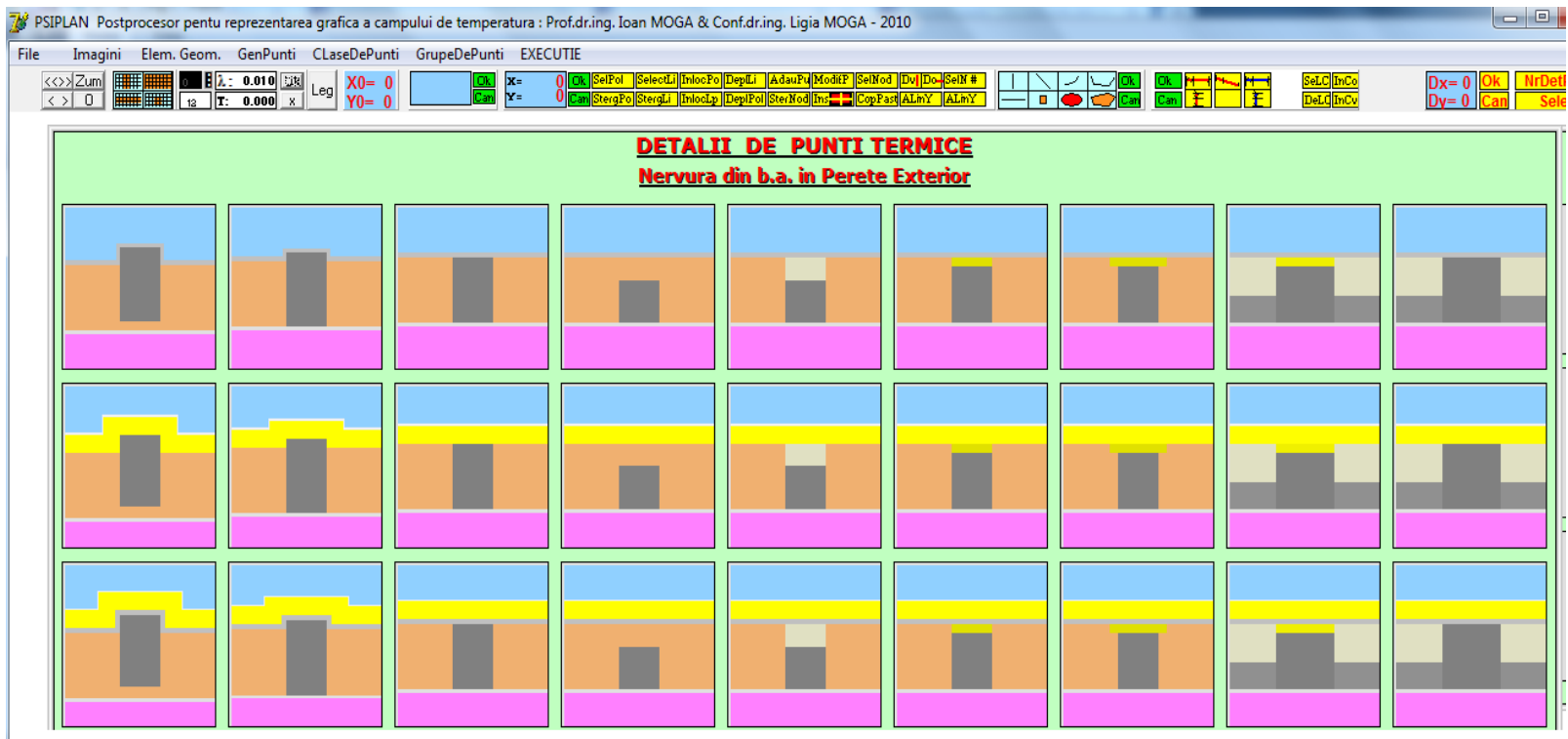

(b)

Fig. 1. Access page for types of thermal bridges (a) and access page of several case studies for a given type (b) 


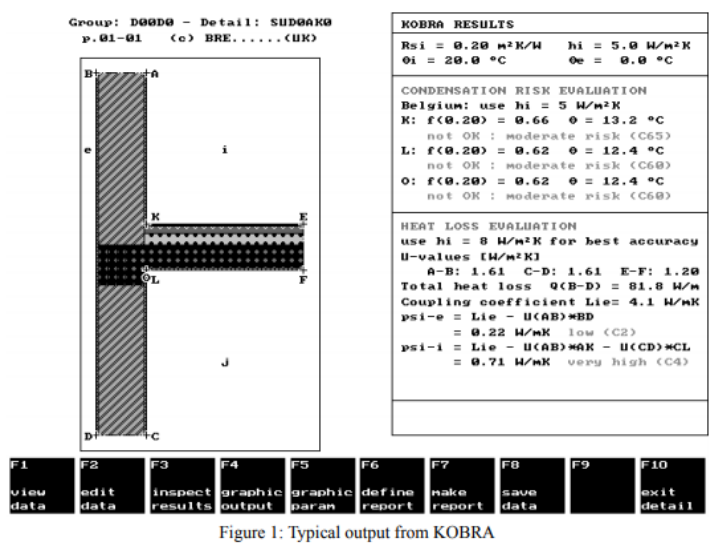

(a)

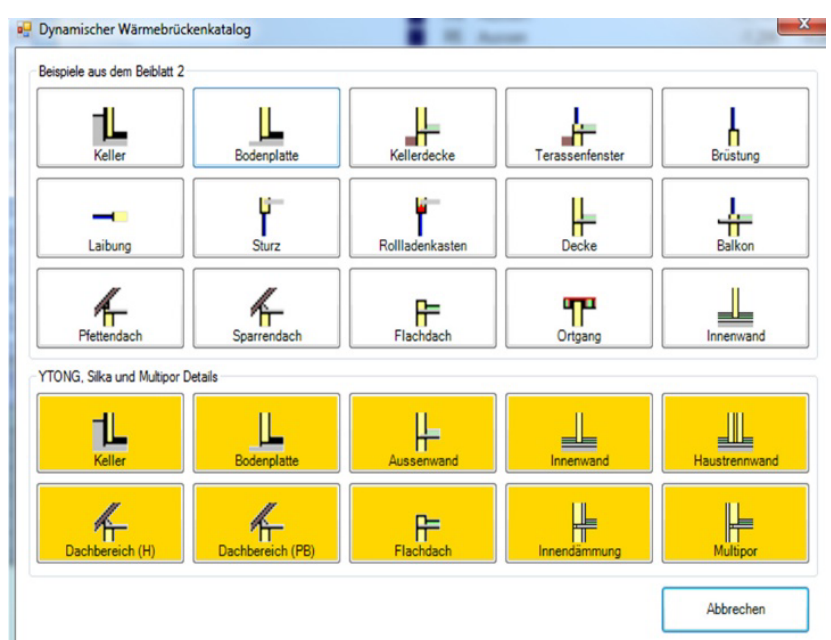

(b)

Fig. 2. (a) Retrieved from EUROKOBRA database [8] and (b) PSI-THERM 3D [www.etu.at]

Each type of thermal bridge includes details starting from a poor thermal design up to optimized solutions. The optimized solutions are the ones used in current practice in designing nZEB, passive houses or similar. The manual of the software will give the proper steps in understanding the usage of the existing database.

In figure 3 are illustrated part of the modelling and simulation steps of the program. Following the identification of the thermal bridge group, the user chooses the most suitable variant for that case of thermal bridge that will be manipulated. The next step requires the adjustment of the thermal characteristics and dimensions for each layer that constitutes the detail. At this stage, new layers can also be added. The boundary conditions for simulation will be chosen. All this criteria will be defined by resetting the dimension lines through the use of the mouse buttons. The thermophysical characteristics will be adjusted with a "click of a button" in the designated area, and the colour describing the material can also be changed. The boundary conditions and the associated colours are modified in the same way. When the detail is final (see fig 3 , case a1), the execution phase of the modelling part is initiated by the user.

At the execution phase, the length of the geometrical model (see fig 3, case a2, b1) will be generated according to the prescription of the European standard [8]. The temperature fields for the extended geometrical model are obtained and the design values are given (see fig 3 , case a2, b2).

Similar initiatives of developing thermal bridges databases were met along the years, but nowadays there is no available online tool for this purpose. For example, the EUROKOBRA database, that contained about 1000 details of all parts of typical European buildings, was made available [16]. The database offered the possibility of assessing steady state 2-D losses and several 3-D losses.

\section{Discussions}

The nZEB context imposed a different design approach in what defines constructive details for the building envelope components. At this moment, the Romanian market is still dominated by massive structures (i.e. reinforced concrete and masonry), and less by lighter constructive approaches (i.e. wood, steel). The design evolution from standard design to nZEB, it starts from known details to optimised versions of those details. 


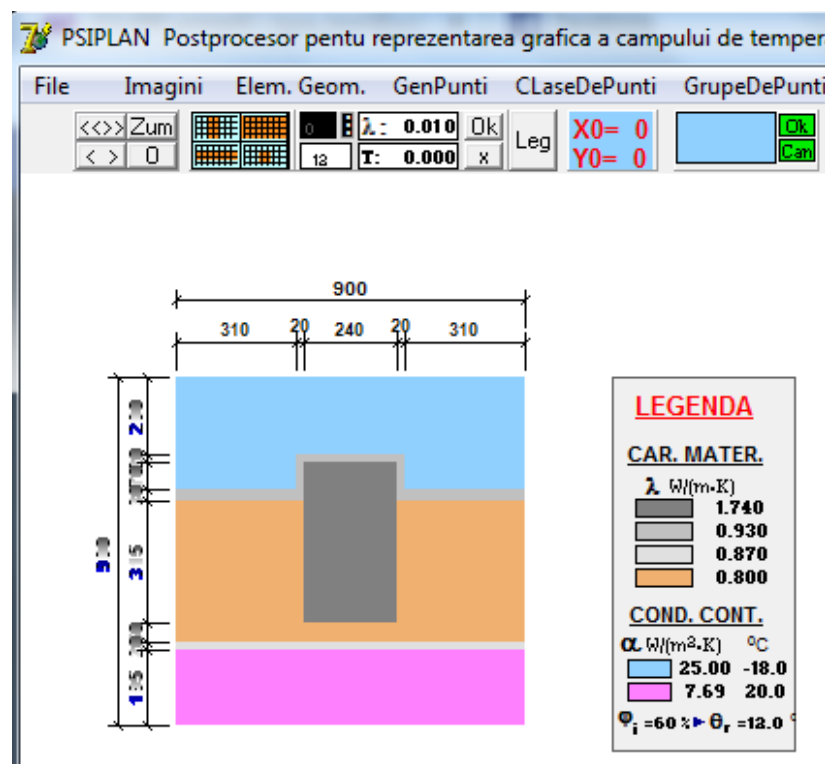

(a.1)

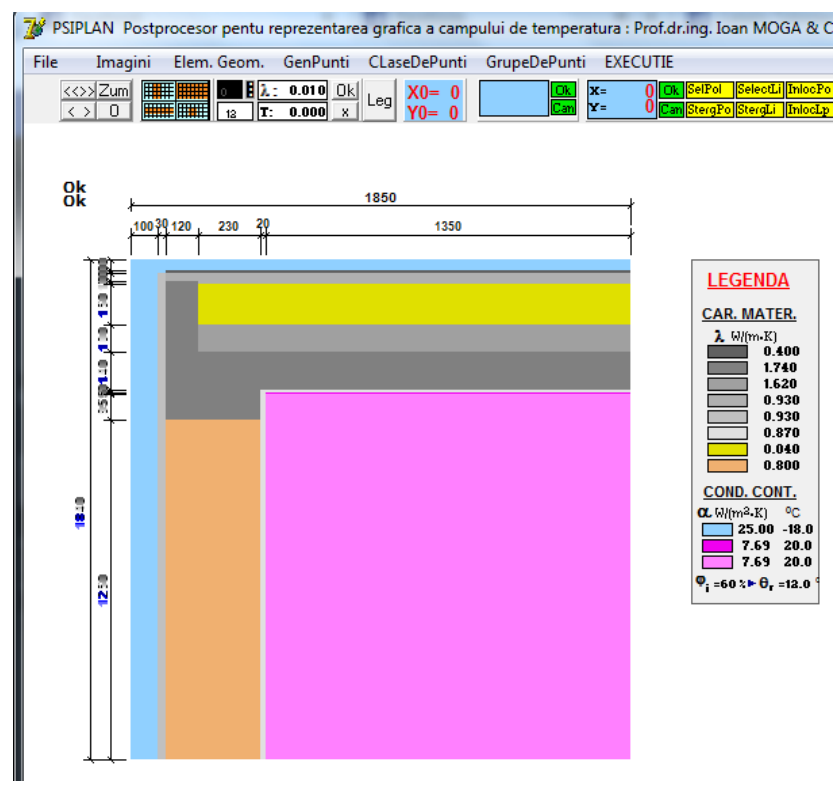

(b.1)

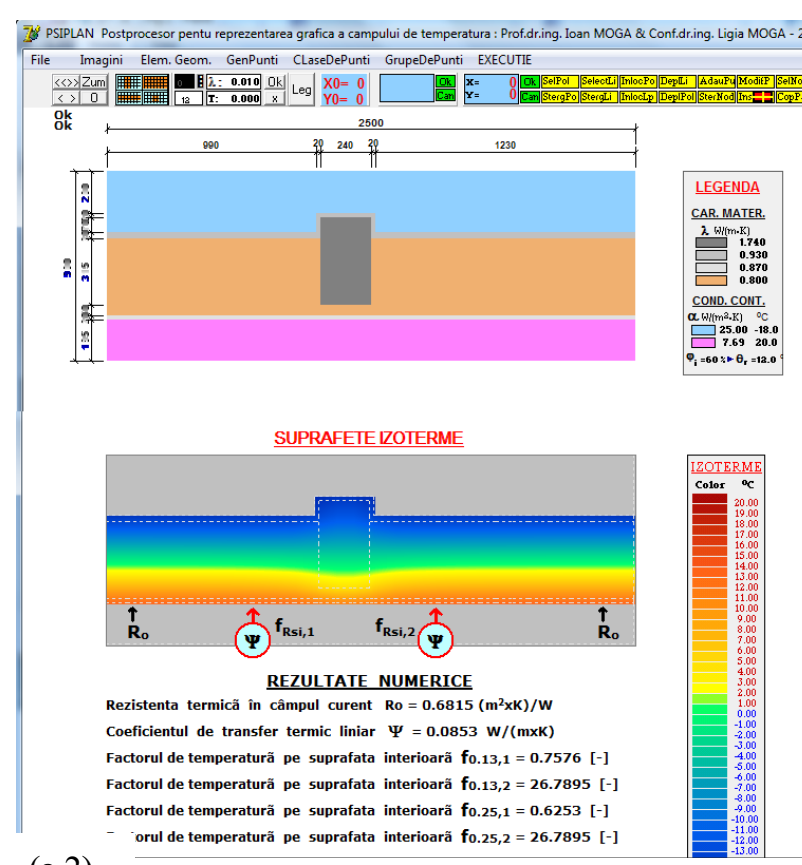

(a.2)

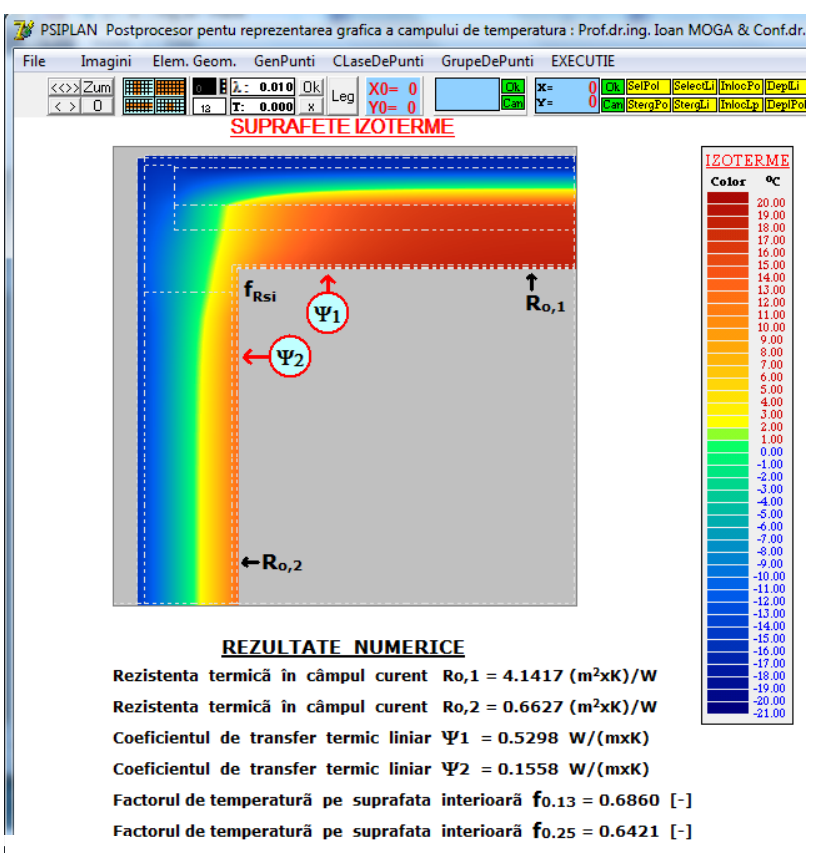

(b.2)

Fig. 3. The geometrical model for (a.1) intermediate column in the exterior wall (b.1) an intersection exterior wall-terrace roof (attic zone); (a.2) and (b.2) temperature fields and numerical results

Practitioners understand the need of changing the design approach, but due to time efficiency and lack of modelling and simulation knowledge, they have a simplified method of addressing the thermal envelope design stage. In many cases, instead of simulating a thermal bridge to get accurate results for what defines the thermal performance of the building envelope, they work with $\Psi$ values defined for similar, but not the same, details existing in catalogues. In other cases, they design constructive solutions based on practical expertise and less based on thermal simulation of the building envelope behaviour. The negative impact in practice, is the fact that the building is underperforming from the thermo-energy point of view, which causes condensation and mould. This approach does not and will never align with the energy and climate framework strategies, and with the nZEB levels imposed for new and existing buildings.

In order to change their design habits, practitioners need a tool that can be accessible from both time and user approach. The software presented in the paper is developed with the aim of addressing this criteria. Additionally, the tool represents a valuable instrument for understanding and learning along the way, the importance of an accurate thermal design. The novelty of the program 
which facilitates the access and understanding to a large varieties of users, can quickly shape the approach towards nZEB design market, as a consequence of modelling more constructive solutions in a quicker and shorter time.

In this regard, the tool contains predefined details that are met in current practice in Romania, from the " 60 s till this moment, but are not limited to those. The user can model other cases, starting from the existing ones. In this fashion, the software responds to both new design and existing buildings retrofit activity. Details specific to other European countries are not yet implemented, but users can manipulate the existing details into completely new ones. Thus, the program by specialist from other countries.

Given the fact that the tool gives the unidirectional thermal resistance $\mathrm{R}$, the linear heat transfer coefficient $\psi$ and the temperature factor $f_{\text {Rsi }}$, one can use this values in software that permits the direct input of this predetermined values. As an example, commercial software like the PHPP software for the design of passive houses or DesignBuilder, require the input of the $\psi$ coefficient calculated by a modelling software or taken from a catalogue. The online software can generate an extract of the results with the help of a text file, which can be further imported in the mentioned software.

\section{Conclusions}

An accurate evaluation of the thermal bridges, is necessary to obtain the thermal behaviour of a building component, behaviour that has a strong impact on the energy performance of the building [17]. It is well known that with higher insulated buildings, the impact of thermal bridges on the energy consumption for heating increases. In the same time thermal bridges modelling and simulation requires advance modelling skills, which require experience in design. The online tool offers a quick instrument in solving typical or complex thermal bridges, in a user friendly way. The tool optimises the time dedicated for elaborating the calculation for the thermo-energy performance of a building.

The online software for thermal bridges assessment is still under development, with the aim of including also details met at curtain walls, metallic structures and other wood structure solutions.

The potential of this tool is directly connected to the European legislation regarding the legislative requirement of increasing the nZEB building stock, at both new and existing buildings. Consequently, beginning with 2021 the nZEB construction market will embrace the use of such tools for the sake of the need in decreasing the time allocated for a project development, but also as a result of changing the design approach towards high performance buildings.

The research was financed by "Susținerea excelenței în cercetarea din domeniul nanotehnologiilor și materialelor avansate" - ExNanoMat Contract nr. 21 PFE-2018.

\section{References}

1. European Parliament, Council of EU, Directive 2010/31/EU on the energy performance of buildings (recast), (2010)

2. European Parliament, Council of EU, Directive 2018/844/EU amending Directive 2010/31/EU on the energy performance of buildings and Directive 2012/27/EU on energy efficiency, (2018)

3. "OVERVIEW | Zero-Energy Buildings: does the definition influence their design and implementation?" accessed at https://www.buildup.eu/en/news/overview-zeroenergy-buildings-does-definition-influence-theirdesign-and-implementation

4. L. Moga, I. Moga, Specific thermal bridges at load bearing masonry buildings - Punţi termice specifice clădirilor cu pereţi structurali din zidărie, U.T. Press, ISBN: 978-973-662-799-6, (2013)

5. ISO 14683, Thermal bridges in building construction - Linear thermal transmittance - Simplified methods and default values, (2017)

6. J. Kosny, E. Kossecka, Multi-dimensional heat transfer through complex building envelope assembrlies in hourly energy simulation programs, Ener. \& Build 34, 445-454, (2002)

7. H. Erhorn-Kluttig, H. Erhorn, Impact of thermal bridges on the energy performance of buildings. Information Paper P148 of the EPBD Buildings Platform, Frahofer Institut for Building Physics, (2009)

8. ISO 10211, Thermal bridges in building construction - Heat flows and surface temperatures - Detailed calculations , (2017)

9. PSIPLAN software. Two-Dimensional HeatTransfer for Buildings Modelling software for calculating linear thermal transmittances values. Building Physics Collective, Technical University of Cluj-Napoca, version 2019

10. SR EN ISO 13790, Thermal performance of buildings. Calculation of the energy necessary for heating, (2005).

11. L. Moga, Analytic Study of Thermal Bridges Met at High Performance Energy Efficient Buildings, 18th International Multidisciplinary Scientific GeoConference SGEM2018, November, (2018)

12. K Kuusk, J. Kurnitski, T. Kalamees, Calculation and compliance procedures of thermal bridges in energy calculations in various European countries, $11^{\text {th }}$ Nordic Symposium on Building Physics, (2017)

13. C107 Norm for thermotechnical calculation of construction elements of buildings, (2005)

14. Annex of the Order 1590, Catalogue of specific thermal bridges for buildings, (2012)

15. Order 2641 regarding the modification and completion of the technical regulation "Methodology for calculating the energy performance of buildings", 
approved by the Order of the Minister of Transport, Construction and Tourism no. 157/2007, (2017)

16. P. Strachan, A. Nakhi, C. Sanders, Thermal bridge assessments.

17. A. Levinskyte, K. Banionis, G. Valdemaras, The Influence of Thermal Bridges for Buildings Energy Consumption of "A" Energy Efficiency Class, Journal of Sustainable Architecture and Civil Engineering, Vol 15, 2, (2016) 